\title{
A SIMPLE FILTERING PROCEDURE FOR REMOVING NEGATIVE VALUES FROM NUMERICAL SOLUTIONS OF THE ADVECTION EQUATION
}

Jerzy Bartnicki

International Institute for Applied Systems Analysis

Laxenburg, A ustria

RR-90-4

April 1990

Reprinted from Environmental Software, 4(4): 187-201.

INTERNATIONAL INSTITUTE FOR APPLIED SYSTEMS ANALYSIS

Laxenburg, Austria 
Research Reports, which record research conducted at IIASA, are independently reviewed before publication. However, the views and opinions they express are not necessarily those of the Institute or the National Member Organizations that support it.

Reprinted with permission from Environmental Software, 4(4): 187-201.

Copyright (C) 1989, Computational Mechanics Publications.

All rights reserved. No part of this publication may be reproduced or transmitted in any form or by any means, electronic or mechanical, including photocopy, recording, or any information storage or retrieval system, without permission in writing from the copyright holder.

Printed by Novographic, Vienna, Austria 


\section{FOREWORD}

Dr. Bartnicki, a meteorologist, has been a member of the Transboundary Air Pollution Project for many years and has contributed much to the construction of the atmospheric transport part of the Regional Acidification and Simulation (RAINS) model. The single most important factor in the dispersion of air pollutants is the advection by the wind. An air pollution model must simulate this process while preserving mass.

In this paper Dr. Bartnicki addresses the problem of avoiding the computation of negative concentrations while using Eulerian algorithms in models of long-range atmospheric transport.

R.W. SHAW, Leader Transboundary Air Pollution Project

B.R. DÖÖS, Leader Environment Program 


\title{
A Simple Filtering Procedure for Removing Negative Values from Numerical Solutions of the Advection Equation
}

\author{
J. Bartnicki* \\ International Institute for Applied Systems Analysis, A-2361 Laxenburg, Austria
}

A simple nonlinear filter which completely removes negative values from the multidimensional numerical fields is presented. This filter conserves total mass (sum of all elements in the field) with accuracy better than $0.0001 \%$. Also, the maxima and shape of the filtered function are unaltered. Basically, the filter can be combined with an arbitrary method used for the numerical solution of the advection equation. Two-dimensional advective tests show that negative values are removed, and short waves are significantly damped with the pseudospectral method. The filtering algorithm is very simple. Additional computational time required by the the filter is about $4 \%$ of that required by the pseudospectral method alone.

Key Words: Digital filter, advection equation, air pollution modeling, pseudospectral method

\section{INTRODUCTION}

The Long Range Transport of Air Pollutants (LRTAP) has been a rather important issue for the last two decades, both in Europe and in the USA. Many LRTAP models, describing transport of different types of pollutants have been developed during that time. Special attention has been paid to sulfur (eg. a special issue of Atmospheric Environment 12, 1978) and also to $\mathrm{NO}_{\mathrm{x}}, \mathrm{O}_{3}, \mathrm{NH}_{\mathrm{x}}$ and heavy metals. Generally, atmospheric transport of air pollutants is described by the partial differential advection-diffusion equation, which can be solved in a Lagrangian (Eliassen and Saltbones [1]; Bottenheim et al. [2]) or an Eulerian (Zlatev et al. [3]; Carmichael [4]) framework. Both methods are, more or less, equivalent in

\footnotetext{
On leave from the Institute for Meteorology and Water Management, Warsaw, Poland.
}

the case of simple two-dimensional LRTAP models. For three-dimensional transport models, especially with complicated nonlinear chemistry, the Eulerian approach has to be used. However, contrary to Lagrangian solution, many of the Eulerian algorithms can produce negative concentrations, which are not only unrealistic, but in addition, can make the numerical solution unstable (Berkowicz, Zlatev - personal communication). In order to avoid this difficulty in the more complicated transport models, it is necessary to use an algorithm which does not generate negative values in the numerical solution of the advection-diffusion equation. One of the possible ways to remove these unwanted negative values is to apply a numerical filter to the solution

The main purpose of this paper is to present a very simple filtering procedure which can be applied to a wide range of 
different numerical methods used to solve basic equations of the LRTAP models.

When solving the advectiondiffusion equation, the diffusive part is less important than the advective part in terms of numerical problems (Zlatev [5]). Also from the physical point of view, in the synoptic scale of motions, the diffusion term is small compared to advection and is even neglected in some LRTAP models (eg. Eliassen and Saltbones [1]). Therefore, only an application of the method to the advection equation is presented in this paper.

\section{Filtering Procedure}

The presence of negative values in the solution is a common phenomenon for the different numerical methods used for solving the advection equation (Soong and Ogura [6], Long and Pepper [7]). According to Forester [8] and Adam [9], this is mainly due to incorrect numerical propagation speed of the shortest waves in the spectrum. They suggested numerical filtering as a relatively easy and efficient way of removing negative values from the solution. Concerning application to the advection equation, the main features of a perfect filtering procedure are: (1) To remove completely negative values, (2) To conserve total mass, (3) To preserve the shape of the function, (4) To preserve the maxima, (5) To be free of short wave noise. Unfortunately, the existing numerical filters (eg. Forester [8], Chapman [10] and the algorithm presented in this paper) do not satisfy all the above requirements.

\subsection{Method}

Despite its simplicity, the multidimensional nonlinear filtering procedure described in this paper fulfills at least some of the conditions mentioned above. In particular, it completely removes negative values, conserves $100 \%$ of the total mass and keeps filtered maxima and shape of the function relatively close to original ones.
The basic idea of the filtering procedure is very simple. When solving the advection equation, negative values are not permitted in the solution. A question arises what to do with the artificial "negative" mass which appears in the numerical solution. Probably, the easiest way of dealing with this problem is to redistribute "negative" mass over positive values in the solution. The next question is: how to do it? Unfortunately, there is no clear mathematical or physical indication for any specific solution. In this situation, we decided to use the simplest possible approach and reduce equally each positive value in the distribution.

More precisely, the concept of the filter can be explained as follows: Let $c_{j}$ be the concentration in the $\mathrm{j}$-th point in the grid system of arbitrary dimension consisting of $N$ points $(j=1, \ldots, N)$. If all $c_{j}$ values are non-negative the filter does not change them. Now, let us assume that the concentration field has $N_{1}$ positive values $\left(c_{j}>0\right), N_{2}$ zero values $\left(c_{j}=0\right)$ and $\mathrm{N}_{3}$ negative values $\left(c_{j}<0\right)$. Obviously

$$
\mathrm{N}_{1}+\mathrm{N}_{2}+\mathrm{N}_{3}=\mathrm{N}
$$

We assume that

$$
\mathrm{M}_{1}>\mathrm{M}_{3}
$$

where

$$
M_{1}=\sum_{j=1}^{N} c_{j}
$$

is the "positive" mass and

$$
M_{3}=\left|\sum_{\substack{j=1 \\ c_{j}<0}}^{N} c_{j}\right|
$$

is the "negative" mass. With the above assumptions the filtering procedure is defined by the following algorithm:

1. Compute the negative mass $\mathbf{M}_{3}$ and check if it is greater than zero. If not, stop. 
2. Compute the number of positive concentrations $\mathrm{N}_{1}$.

3 . Check the sign of the concentration $c_{j}$ for $j=1, \ldots, N$

(a) If $c_{j}>0$, subtract the negative mass divided by the number of positive concentrations: $c_{j}:=c_{j}-\frac{M_{3}}{N_{1}}$

(b) If $\mathrm{c}_{\mathrm{j}}=0$, do nothing.

(c) If $c_{j}<0$, set it to zero: $c_{\mathrm{j}}:=0$.

4. Go to 1 .

The algorithm presented above is convergent (Bartnicki [11]) and typically only two iterations are necessary to remove all negative values. The listing of the FORTRAN subroutine for the filter is included in the Appendix.

\subsection{Examples}

The filtering procedure defined in the previous section is illustrated by application to two different concentration fields, both including negative values. In the first case, the digital filter was applied to a one-dimensional distribution in the grid system consisting of 11 points. The initial distribution shown in Figure (1a) is typical for the intermediate solution of the advection equation with a "delta" function (concentrations at all points except one are equal to zero) as an initial condition. Two negative values of concentration are present in the distribution: -4 at point number 4 and -5 at point number 8. After the first iteration (Figure 1b) only one negative value remains: -0.8 at point number 11 . The second and final iteration (Figure 1c) gives a distribution without negative values. The maximum is slightly lower: 13 instead of 15 but the shape of the final distribution is quite close to the initial one (Figure 1d). From Figure 1d, it can be also seen that the short waves present in the initial distribution have been removed from the final one.
In the second example, a twodimensional concentration field was used. The initial distribution (Fig. 2a) was designed as a superposition of a rectangular block and randomly generated noise with a range $\pm 10 \%$ of the rectangular block height. The initial distribution obtained in this way (Fig. 2a) is a difficult test case for a filtering procedure because it includes both sharp gradients and short wave noise. The result of the filtering is shown in Fig. 2b. All negative values are completely removed from the initial distribution. In addition, the noise is smoothed everywhere, except for the rectangular block where the filter does not touch it. Again, the final shape and the maximum of the function are relatively close to the initial ones.

It should be stressed that the main purpose of the algorithm presented in this paper is to remove negative values from the numerical field. Reduction of the noise can be achieved more efficiently by many other filters specially designed for this task. For example, Pepper et al. [12] suggest two effective algorithms which can be applied to the numerical solution of the advection equation: an implicit filter and a filter with a high order dissipative term (Raymond and Garder [13]). In some cases, oscillatory solutions of the advection equation can not be considered as a noise at all (Gresho and Lee [14]) because they carry some important messages indicating, for example, wrongly posed boundary conditions or too coarse mesh for the problem. In these cases reexamination of the method can improve solution better than the application of a filter.

It is difficult to find a general response function or a filter factor for the algorithm presented here, because it is only sensitive to the negative values. It means that all positive waves are transmitted without any damping. On the other hand, the waves (or arbitrary functions) symmetrical to the $\mathrm{x}$-axis are completely removed from the output. In all other cases, when both positive and negative values are present in the input, maxima of the filtered function are slightly reduced which may introduce 

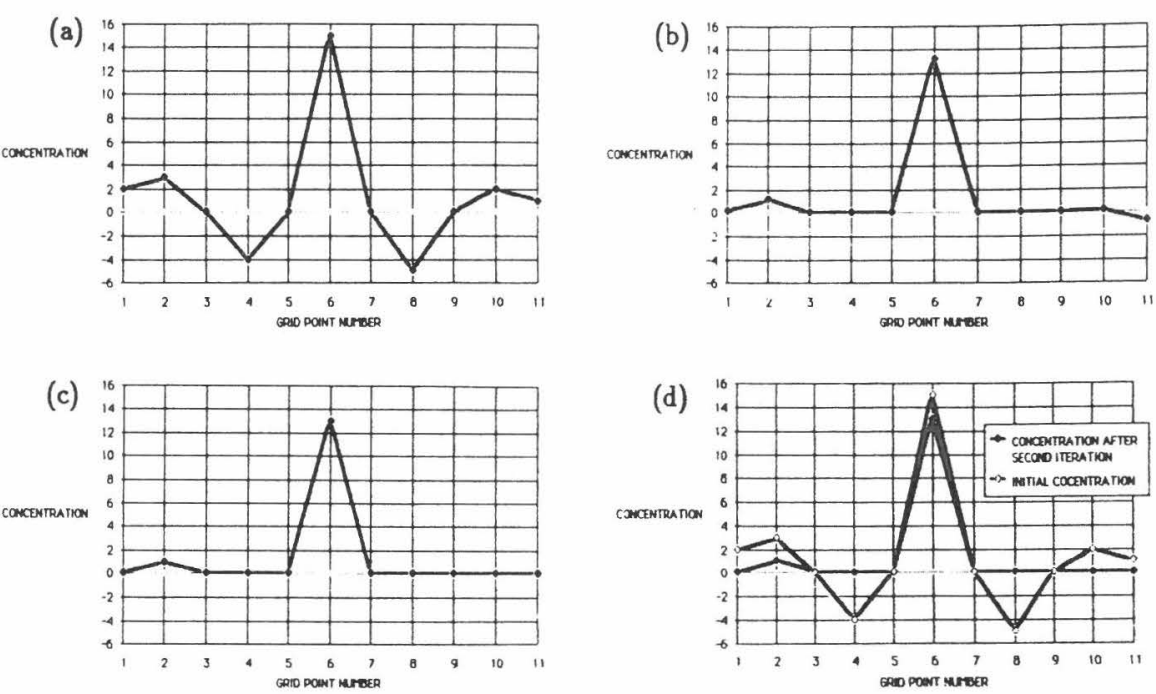

Figure 1. One dimensional test for the filtering procedure:
(a) Initial distribution of the concentration.
(b) Distribution after first iteration.
(c) Distribution after second and final iteration.
(d) Comparison of initial and final distribution of the concentration.
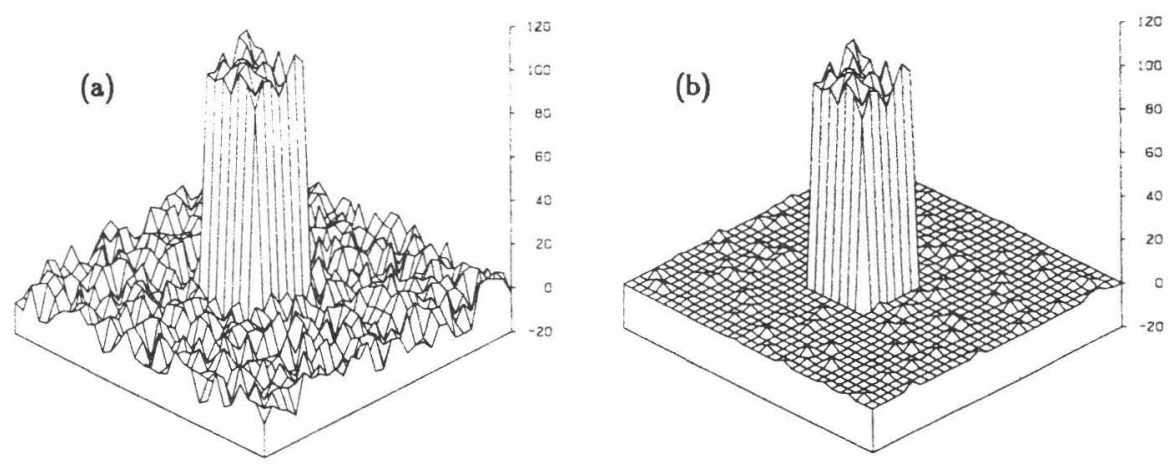

Figure 2. Two dimensional test for the filtering procedure:

(a) Initial distribution of the concentration.

(b) Distribution after second and final iteration. 
numerical diffusion when solving the advection equation. However, this artificial diffusion is relatively small, which will be shown later in several examples. A basic feature of the algorithm illustrated on two above examples is the conservation of mass, which can be expressed as

$$
\mathrm{M}_{1}-\mathrm{M}_{3}=\text { const. }
$$

This algorithm is simple in its numerical realization and fast in the execution. It can be efficiently applied in two ways to a wide range of methods used for the numerical solution of the advection equation:

(1) as a filter to remove negative values at each time step,

(2) as a filter to maintain positive values in the final numerical solution.

The first application is very important when negative values in the numerical solution are not permitted at all. The second when they are prohibited only in the final numerical solution. In this paper, both applications will be compared in the numerical tests. As an example of the first possible application of the filter, the filtering procedure will be combined with an accurate pseudo-spectral method.

\section{POSITIVE DEFINITE VER- SION OF THE PSEUDOSPEC- TRAL METHOD}

Among many different methods used for the numerical solution of the advection equation, the spectral (Orszag [15]) and pseudospectral (Gottlieb and Orszag (16)) approach are relatively efficient and accurate. The accuracies of these methods are better when compared with finite difference methods (Orszag [17]), and also with other methods (Long and Pepper [7], Chock [18]). Another advantage of spectral methods is a simple mathematical formulation which makes them convenient for practical applications, especially when using numerical Fast Fourier Transform (FFT) (Cooley and Tukey [19]). Spectral and pseudospectral methods have been successfully applied to the air pollution transport models by Christensen and Prahm [20], Wengle et al. [21], Zlatev et al. [22], Zlatev et al. [3] and Zlatev [5]. Unfortunately, these accurate methods can produce negative values during the numerical solution of the advection equation. This problem can be solved by combining pseudospectral solution with the filtering procedure described in the previous section. This combined method, which is called "Positive Definite version of the Pseudo-Spectral (PDPS) method", completely eliminates negative values from the solution of the advection equation.

\subsection{Problem Formulation}

The multidimensional advection equation to be solved has the following form:

$$
\frac{\partial c}{\partial t}+\sum_{j=1}^{N} u_{j} \frac{\partial c}{\partial x_{j}}=0
$$

where $c=c(x, t)$ is the concentration (which could be arbitrarily scalar) assumed to be non-negative, $u_{j}=u_{j}(x, t)$ is the $j$-th velocity component, $(x, t)=\left(x_{1}, \ldots, x_{N}, t\right)$ is the space and time coordinates.

The numerical method presented in this paper involves two basic steps at each time step when solving equation (5):

(1) The pseudospectral method is applied to equation (5) at time $t$, and a solution is achieved. This solution can contain negative concentration values.

(2) The filtering procedure removes all negative values of the concentration and obtains a new solution at time $t+\Delta t$.

Let $c^{m}=c(x, m \Delta t)$ be the concentration field with periodic boundary conditions at time $m \Delta t$. We are looking for the concentration $c^{m+1}=c(x,(m+1) \Delta t)$ at time $(m+1) \Delta t$ in the uniform mesh of size $\mathrm{L}_{1} \times \mathrm{L}_{2} \times \ldots \times \mathrm{L}_{\mathrm{N}}$ where the location of the mesh points is given by: 
$\mathrm{x}_{\mathrm{j}}=\mathrm{l}_{\mathrm{j}} \Delta \mathrm{x}_{\mathrm{j}} ; \quad \mathrm{l}_{\mathrm{j}}=0,1,2, \ldots, \mathrm{L}_{\mathrm{j}}-1$

where

$$
\Delta x_{j}=\frac{2 \pi}{L_{j}}
$$

for $\mathrm{j}=1,2, \ldots, \mathrm{N}$.

The pseudospectral method can be represented by an operator $\hat{\mathrm{P}}$ which, when applied to the discrete concentration field $c^{m}$ at time $m \Delta t$, produces the concentration $\tilde{c}^{\mathrm{m}+1}$ at time $(\mathrm{m}+1) \Delta \mathrm{t}$ :

$$
\tilde{\mathrm{c}}^{\mathrm{m}+1}=\hat{\mathbf{P}}\left(\mathrm{c}^{\mathrm{m}}\right)
$$

The concentration $\tilde{c}^{\mathrm{m}+1}$ can still include negative values. The filtering procedure can be represented by the operator $\hat{F}$ which transforms $\tilde{c}^{\mathrm{m}+1}$ to $\mathrm{c}^{\mathrm{m}+1}$ containing only non-negative values :

$$
\mathrm{c}^{\mathrm{m}+1}=\hat{\mathrm{F}}\left(\tilde{\mathrm{c}}^{\mathrm{m}+1}\right)
$$

Thus, the positively defined pseudospectral method can be defined as:

$$
\mathrm{c}^{\mathrm{m}+1}=\hat{\mathrm{F}} \cdot \hat{\mathrm{P}}\left(\mathrm{c}^{\mathrm{m}}\right)
$$

In principle the operator $\hat{\mathbf{P}}$ can represent other methods (this will be a subject of a separate paper), in addition to the pseudospectral method. However, because of its simplicity and accuracy, the pseudospectral approximation is rather efficient for the numerical solution of equation (5). In the next section we briefly review this method.

\subsection{Pseudospectral Solution}

The pseudospectral approach developed by Gazdag [23] has been chosen as the operator $\hat{\mathrm{P}}$, because of its high accuracy. The principle of Gazdag's method is to approximate the time derivatives by a truncated Taylor series, and then replace the time derivatives by the space derivative terms which are computed using the spectral method. Mathematically, the method can be described as follows: Assuming that we know the concentration $c^{\mathrm{m}}$ at time $\mathrm{m} \Delta \mathrm{t}$, the concentration $c^{m+1}$ at the next time step $(m+1) \Delta t$ can be approximated by the truncated Taylor series

$$
\begin{aligned}
c^{\mathrm{m}+1} & =c^{\mathrm{m}}+\frac{\partial c^{\mathrm{m}}}{\partial \mathrm{t}} \Delta \mathrm{t}+\frac{\partial^{2} c^{\mathrm{m}}}{\partial \mathrm{t}^{2}} \frac{\Delta \mathrm{t}^{2}}{2 !}+ \\
& +\frac{\partial^{3} c^{\mathrm{m}}}{\partial \mathrm{t}^{3}} \frac{\Delta \mathrm{t}^{3}}{3 !}+\cdots+\frac{\partial^{\mathrm{P}} c^{\mathrm{m}}}{\partial \mathrm{t}^{\mathrm{p}}} \frac{\Delta \mathrm{t}^{\mathrm{p}}}{\mathrm{p} !}
\end{aligned}
$$

Following Gazdag [23], the time derivatives of $c$ can be expressed in terms of the space derivatives of $c$ and $u_{j} b y$ making use of equation (5):

$$
\begin{gathered}
\frac{\partial c}{\partial t}=-\sum_{j=1}^{N} u_{j} \frac{\partial c}{\partial x_{j}} \\
\frac{\partial^{2} c}{\partial t^{2}}=-\sum_{j=1}^{N}\left\{\frac{\partial u_{j}}{\partial t} \frac{\partial c}{\partial x_{j}}+u_{j} \frac{\partial}{\partial x_{j}} \frac{\partial c}{\partial t}\right\} \\
\cdot \\
\frac{\partial^{l+1} c}{\partial t^{1+1}}=-\sum_{j=1}^{N} \sum_{r=0}^{i}(l) \frac{\partial^{r} u_{j}}{\partial t^{r}} \frac{\partial}{\partial x_{j}}\left(\frac{\partial^{1-r} c}{\partial t^{1-r}}\right)
\end{gathered}
$$

The superscript $m$ has been omitted in the above equations for convenience. Equations (12-14) show how to compute any order time derivative of $c$ from the lower order time derivatives of $u_{j}$ and $c$. The first order time derivative of $c$ can be computed directly from the basic advection equation. It remains only to compute space derivatives of $c$. This is done with the spectral method. Denoting the set of all grid points (Equations 6-7) by $\mathbf{R}$, the finite Fourier transform $\mathrm{C}$ of $\mathrm{c}$ can be written as

$$
C(\mathbf{k}, \mathrm{t})=\frac{1}{\mathrm{~L}_{1} \mathrm{~L}_{2} \cdots \mathrm{L}_{\mathrm{N}}} \sum_{\mathbf{x} \in \mathrm{R}} \mathrm{c}(\mathbf{x}, \mathrm{t}) \mathrm{e}^{-\mathrm{i} \mathbf{k} \cdot \mathbf{x}}
$$


where $\mathrm{i}=\sqrt{-1}$ and $\mathbf{k}$ is the wave vector

$$
\mathbf{k}=\left(\mathbf{k}_{1}, \ldots, \mathbf{k}_{\mathrm{j}}, \ldots, \mathrm{k}_{\mathrm{N}}\right)
$$

whose components assume integer values within the limits

$$
-\mathrm{K}_{\mathrm{j}}<\mathrm{k}_{\mathrm{j}} \leq \mathrm{K}_{\mathrm{j}} ; \quad \mathrm{K}_{\mathrm{j}}=\frac{\mathrm{L}_{\mathrm{j}}}{2}
$$

From $C(k, t)$ the partial derivatives of $c(x, t)$ can be computed as

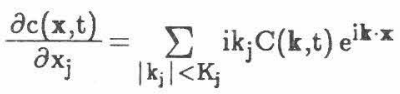

The numerical computation of the space derivatives described by Equations (15-18) can be carried out sufficiently fast by the use of the numerical Fast Fourier Transform (FFT, Cooley and Tukey [19]). According to Gazdag [23] such computation gives very accurate results which prompt him to call it the Accurate Space Derivative (ASD) method. Stability condition of the ASD method depends on the order of expansion $p$ in equation (11) and has following form:

$$
\left|\sum_{1=0}^{p} \frac{(i \varphi)^{l}}{l !}\right| \leq 1
$$

where

$$
\varphi=-\mathbf{k} \cdot \mathbf{v} \Delta t
$$

For example, stability condition (19) is satisfied by ASD schemes of order $3,4,7$ and 8 . In case of the third order expansion, the Courant limit associated with the pseudospectral method is 0.5 .

In the next paragraph accuracy of the PDPS method will be tested by comparing the numerical and analytical solutions of the advection equation.

\section{ADVECTIVE TEST}

In order to check the application of the filtering procedure, an analytical solution of the advective equation was compared with the numerical one. An artificial velocity field was used in the analytical test, in which an initial condition rotated around the axis without changing its shape. Three different initial conditions were chosen: cone, rectangular block and "delta" function. The test was performed both for the Positive Definite Pseudo-Spectral (PDPS) method, and Pseudo-Spectral (PS) approach described in the previous paragraph. In addition, the filtering procedure was applied to the pseudospectral results after ten rotations. This FPS (Filtered Pseudo-Spectral) solution was compared with PDPS and PS results.

\subsection{Basic Equation}

The equation describing the rotation of the "frozen" initial condition has been frequently used for testing numerical methods (Orszag [15], Gazdag [23] Long and Pepper [7], Christensen and Prahm $[20])$. It has the following form:

$$
\frac{\partial c}{\partial t}-\omega y \frac{\partial c}{\partial x}+\omega x \frac{\partial c}{\partial y}=0
$$

where $\omega$ is angular velocity

$$
\omega=\frac{2 \pi}{T}
$$

and $T$ is the period of rotation. Equation (21) was solved numerically in a grid consisting of 32 nodes each in the $x$ - and $y$ directions. The time step was $\frac{T}{400}$, which meant that one full revolution required 400 time steps. The analytical and numerical solutions were compared after each of 10 rotations. In addition, several parameters were computed during each run. Namely:

(1) Mass conservation (in \%) - $\mathrm{M}$ 


$$
M=\frac{\sum_{i=1}^{32} \sum_{j=1}^{32} c(i, j)}{\sum_{i=1}^{32} \sum_{j=1}^{32} c_{0}(i, j)} \times 100
$$

where $c_{0}(i, j)$ is the initial concentration

(2) Conservation of the square of the mass (in \%) - SM

$$
S M=\frac{\sum_{i=1}^{32} \sum_{j=1}^{32} c^{2}(i, j)}{\sum_{i=1}^{32} \sum_{j=1}^{32} c_{0}^{2}(i, j)} \times 100
$$

(3) Minimum of $c(i, j)-$ MIN

(4) Maximum of $c(i, j)-M A X$

(5) Maximum error - MER

$$
\operatorname{MER}=\max _{i, j}\left(c(i, j)-c_{0}(i, j)\right)
$$

The norm given by equation (24) describes the amplification of the waves in the solution. Other possible norms, for example $\sum_{i, j}[c(i, j)]^{4}, \quad \sum_{i, j}\left(\left.\frac{\partial c}{\partial x}\right|_{i, j}\right)^{2}$, were not taken into account because they are less important for the application of the method to the LRTAP models. All tested initial conditions had the same maximum equal to 100 . For both PDPS and PS method periodic boundary conditions were applied during the numerical solution of Equation (21).

\subsection{Cone Shape Initial Condition}

The advection Equation (21) with the "cone" shape initial condition (Figure $3 a$ ) is a standard analytical test and has been applied to many numerical methods. In the grid system, the "cone" shape is defined as:

$$
c_{0}(i, j)= \begin{cases}100 \cdot(1-r / 4) & \text { if } r \leq 4 \\ 0 & \text { if } r>4\end{cases}
$$

$$
\begin{gathered}
r=\sqrt{(i-8)^{2}+(j-16)^{2}} \\
i, j=1, \ldots, 32
\end{gathered}
$$

The numerical solutions after ten rotations are shown in Figures (3b), (3c) and (3d) for the PDPS, PS and FPS methods, respectively. The difference in shapes is small and all numerical solutions are quite close to the analytical one. However, negative values appear only in the PS solution, reaching -1.7 as the lowest value.

The full results of the numerical PDPS and PS solutions after each of ten rotations are presented in Table 1.

For both, PDPS and PS method, the total mass, defined by Equation (22), is conserved in $100 \%$ during the entire run with an accuracy better than $0.0001 \%$. The square of the mass, defined by Equation (23), is well conserved by the PS method (94.1\% after 10 rotations) and slightly worse by the PDPS method (92.6\% after 10 rotations). In the latter case the square of the mass decreases rapidly during the first rotation and then stays almost at the same level.

The analytical maximum is equal to 100; the numerical values yield 91.4 for the PDPS method and 94.0 for the PS solution, after ten rotations. For both methods the maximum decreases mainly during the first rotation and then stays at the same level.

For both PDPS and PS methods, the maximum error, defined by Equation (24), occurs at the top of the cone. After ten rotations, it is slightly higher for PDPS method (-8.5) than for the PS ( $-6.0)$.

Inspection of Table 1 and Figure 3 indicates that for both the PDPS and PS methods as well as for FPS algorithm, numerical results are very close to the analytical solution. It also indicates that for more accurate numerical methods, the advection Equation (21) with the cone initial condition is an easy test to pass. 
Table 1. PDPS vs. PS solution of the advection equation with cone initial condition.

\begin{tabular}{|c|cc|cc|cc|cc|c|c|}
\hline \multirow{2}{*}{$\begin{array}{c}\text { Rotation } \\
\text { No }\end{array}$} & \multicolumn{2}{|c|}{ Mass } & \multicolumn{2}{c|}{ Mass squared } & \multicolumn{2}{c|}{ Maximum } & \multicolumn{2}{c|}{ Minimum } & \multicolumn{2}{c|}{ Max. error } \\
\cline { 2 - 11 } & PDPS & PS & PDPS & PS & PDPS & PS & PDPS & PS & PDPS & PS \\
\hline $\mathbf{1}$ & 100.0 & 100.0 & 94.2 & 97.9 & 96.5 & 98.0 & 0.0 & -0.6 & -3.8 & -2.1 \\
2 & 100.0 & 100.0 & 93.7 & 97.7 & 95.2 & 97.1 & 0.0 & -0.9 & $-\mathbf{4 . 8}$ & -2.9 \\
3 & 100.0 & 100.0 & 93.5 & 96.6 & 94.4 & 96.3 & 0.0 & -1.1 & $-\mathbf{5 . 5}$ & -3.7 \\
4 & 100.0 & 100.0 & 93.3 & 96.5 & 93.8 & 96.3 & 0.0 & -1.3 & -6.1 & -3.7 \\
5 & 100.0 & 100.0 & 93.0 & 95.5 & 93.3 & 95.7 & 0.0 & -1.5 & -6.7 & -4.3 \\
6 & 100.0 & 100.0 & 92.9 & 95.4 & 92.9 & 95.2 & 0.0 & -1.7 & $-\mathbf{7 . 1}$ & -4.8 \\
7 & 100.0 & 100.0 & 92.8 & 94.3 & 92.4 & 94.9 & 0.0 & -1.7 & -7.5 & -5.1 \\
8 & 100.0 & 100.0 & 92.8 & 94.2 & 92.1 & 94.3 & 0.0 & -1.7 & -7.9 & -5.7 \\
9 & 100.0 & 100.0 & 92.7 & 94.1 & 91.8 & 94.2 & 0.0 & -1.7 & -8.2 & -5.8 \\
10 & 100.0 & 100.0 & 92.6 & 94.1 & 91.4 & 94.0 & 0.0 & -1.6 & -8.5 & -6.0 \\
\hline
\end{tabular}
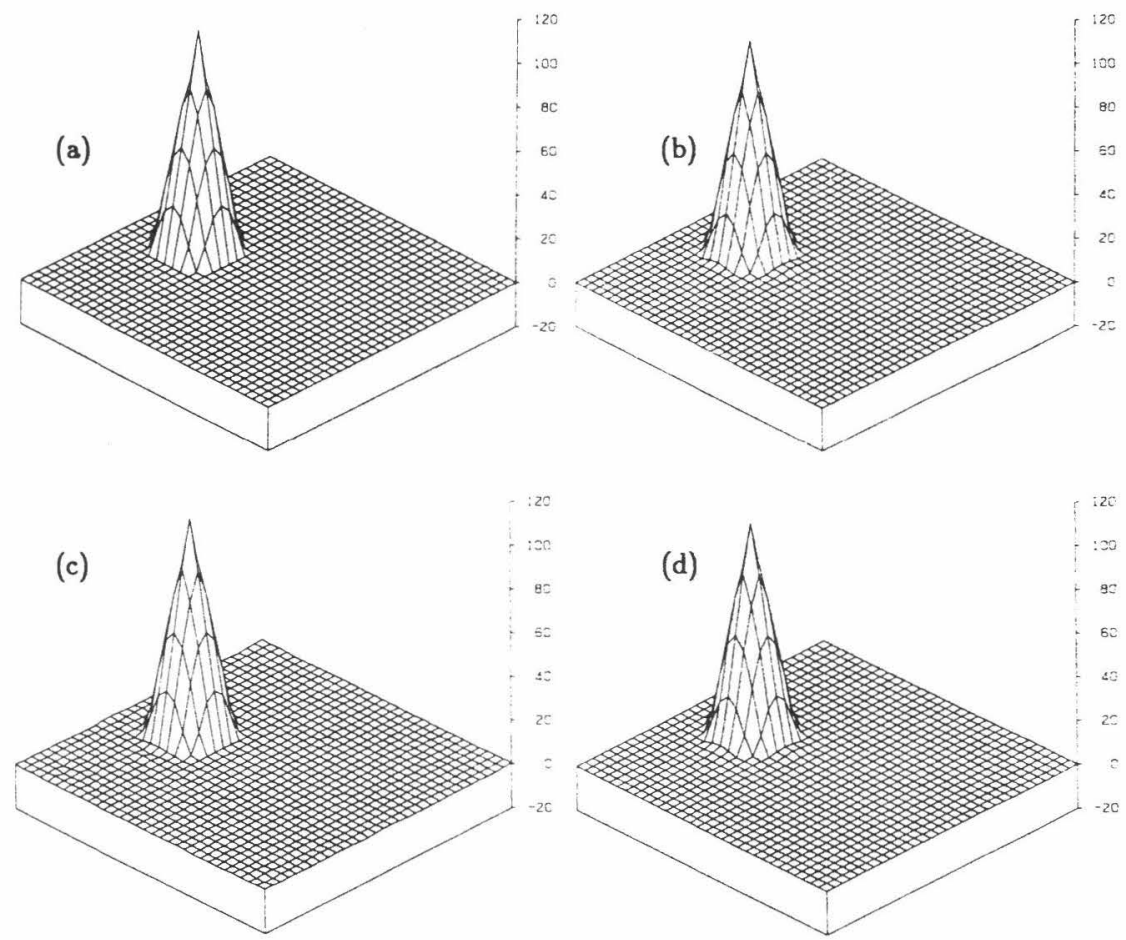

Figure 3. Shape of the cone after 10 rotations:
(a) Analytical solution.
(b) PDPS method.
(c) PS method.
(d) FPS method. 


\subsection{Rectangular Block Initial Con- dition}

The "Rectangular Block" initial condition is shown in Figure 4a. It is defined in the numerical grid as:

$$
\begin{gathered}
c_{0}(i, j)= \begin{cases}100 & \text { if } 5 \leq i \leq 11 \text { and } 13 \leq j \leq 19 \\
0 & \text { otherwise }\end{cases} \\
i, j=1, \ldots, 32
\end{gathered}
$$

The numerical solutions after ten rotations are shown in Figures (4b), (4c) and (4d), for the PDPS, PS and FPS method, respectively. In this case, the differences between analytical and numerical solutions are greater than for the cone shape initial condition. However, also in this case, the initial shape is kept quite well during the entire run. An important advantage of the PDPS method over the PS approach is the absence of short wave noise.

Parameters of the numerical solutions after each rotation are presented in Table 2. The total mass is conserved in $100 \%$ for both methods during the entire run.

The square of the mass is better conserved by the PS method ( $95.7 \%$ after ten rotations) than by the PDPS method (69.4\% after ten rotations). Again, as in the case of the cone shape, the square of the mass decreases mainly during the first rotation $(71.4 \%)$ for PDPS, and then remains at the same level (around 70\%).

The negative concentrations appear only in the PS solution, varying from -9.28 after the third rotation to $\mathbf{- 1 3 . 0 7}$ after the tenth rotation. The negative values produced by the PS method are significantly lower (more negative) for the rectangular block initial condition than for the cone initial condition because of the sharp gradient at the edges of the block.

The maximum for the PDPS method increases to 105.6 after the first rotation and then decreases continuously to 101.0 after ten rotations. The maximum for the PS method is about $20 \%$ higher than the analytical value. It reaches 120.16 after the fourth rotation and then declines afterwards.

For both, the PDPS and PS methods the maximum errors are much higher compared to the cone initial condition. In the case of PDPS, the maximum error increases rapidly to 38.9 after the first rotation, and then slowly goes to 47.1 after ten rotations. In the case of the PS method, maximum error increases to 38.8 after seven rotations. For the rectangular block initial conditions, the maximum error occurs mainly at the points with the highest gradient, at the edges of the block.

\section{4. "Delta" Initial Condition}

The "delta" function (Figure 5a) is probably the most difficult test case for the numerical methods. In LRTAP models "delta" can represent emissions from one small country, for example Luxembourg in the EMEP MSC-W model (Dovland and Saltbones [24]). It can also represent single emission sources in other smaller-scale models. In the numerical grid system "delta" is defined as follows:

$$
\begin{aligned}
c_{0}(i, j) & = \begin{cases}100 & x=8 \text { and } y=16 \\
0 & \text { all other } i \text { and } j\end{cases} \\
i, j & =1, \ldots, 32
\end{aligned}
$$

The numerical solutions after ten rotations are shown in Figures (5b), (5c) and (5d) for the PDPS, PS and FPS methods, respectively. For all cases, the differences between analytical and numerical solutions are relatively large. Again, like in the case of rectangular block initial condition, the negative concentrations (9.7 after 7 rotations) and short waves present in the PS solution are removed from PDPS solution. For both methods, the total mass is again conserved in $100.0 \pm 0.0001 \%$ during the entire run. 
Table 2. PDPS vs. PS solution of the advection equation with rectangular block initial condition.

\begin{tabular}{|c|c|c|c|c|c|c|c|c|c|c|}
\hline \multirow{2}{*}{$\begin{array}{c}\text { Rotation } \\
\text { No }\end{array}$} & \multicolumn{2}{|c|}{ Mass } & \multicolumn{2}{|c|}{ Mass squared } & \multicolumn{2}{|c|}{ Maximum } & \multicolumn{2}{|c|}{ Minimum } & \multicolumn{2}{|c|}{ Max. error } \\
\hline & PDPS & PS & PDPS & PS & PDPS & PS & PDPS & PS & PDPS & PS \\
\hline 1 & 100.0 & 100.0 & 71.4 & 98.9 & 105.6 & 108.9 & 0.0 & -12.3 & -38.9 & -15.0 \\
\hline 2 & 100.0 & 100.0 & 70.8 & 98.2 & 104.2 & 114.1 & 0.0 & -10.4 & -40.7 & -25.7 \\
\hline 3 & 100.0 & 100.0 & 70.4 & 97.7 & 103.4 & 115.5 & 0.0 & -9.3 & -42.2 & -29.3 \\
\hline 4 & 100.0 & 100.0 & 70.2 & 97.3 & 102.8 & 120.2 & 0.0 & -10.2 & -43.4 & -35.2 \\
\hline 5 & 100.0 & 100.0 & 70.0 & 96.9 & 102.4 & 117.9 & 0.0 & -9.5 & -44.4 & -34.8 \\
\hline 6 & 100.0 & 100.0 & 69.8 & 96.6 & 102.0 & 117.9 & 0.0 & -10.3 & -45.2 & -35.4 \\
\hline 7 & 100.0 & 100.0 & 69.7 & 96.4 & 101.7 & 118.9 & 0.0 & -11.2 & -45.8 & -38.8 \\
\hline 8 & 100.0 & 100.0 & 69.6 & 96.1 & 101.5 & 114.9 & 0.0 & -11.5 & -46.3 & -35.5 \\
\hline 9 & 100.0 & 100.0 & 69.5 & 95.9 & 101.4 & 113.4 & 0.0 & -10.6 & -46.7 & -34.7 \\
\hline 10 & 100.0 & 100.0 & 69.4 & 95.7 & 101.0 & 114.4 & 0.0 & -13.1 & -47.1 & -35.0 \\
\hline
\end{tabular}
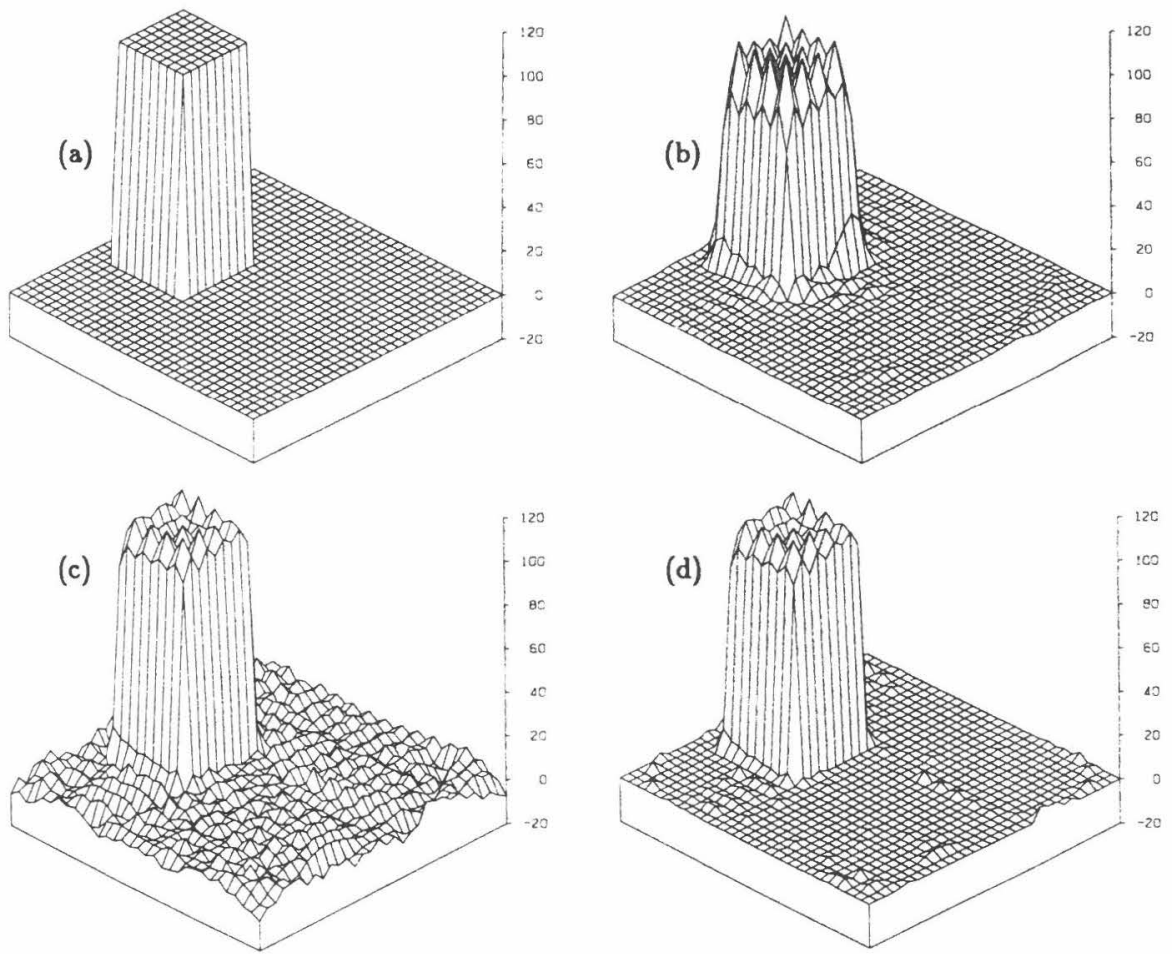

Figure 4. Shape of the rectangular block after 10 rotations:
(a) Analytical solution.
(b) PDPS method.
(c) PS method.
(d) FPS method. 
Table 3. PDPS vs. PS solution of the advection equation with "delta" initial condition.

\begin{tabular}{|c|c|c|c|c|c|c|c|c|c|c|}
\hline \multirow{2}{*}{$\begin{array}{c}\text { Rotation } \\
\text { No }\end{array}$} & \multicolumn{2}{|c|}{ Mass } & \multicolumn{2}{|c|}{ Mass squared } & \multicolumn{2}{|c|}{ Maximum } & \multicolumn{2}{|c|}{ Minimum } & \multicolumn{2}{|c|}{ Max. error } \\
\hline & PDPS & PS & PDPS & PS & PDPS & PS & PDPS & PS & PDPS & PS \\
\hline 1 & 100.0 & 100.0 & 10.4 & 89.1 & 19.5 & 70.2 & 0.0 & -7.4 & -86.5 & -29.7 \\
\hline 2 & 100.0 & 100.0 & 9.7 & 81.6 & 18.4 & 68.7 & 0.0 & -9.2 & -86.6 & -31.3 \\
\hline 3 & 100.0 & 100.0 & 9.4 & 76.0 & 17.9 & 61.4 & 0.0 & -8.3 & -86.8 & -38.6 \\
\hline 4 & 100.0 & 100.0 & 9.2 & 76.1 & 17.6 & 61.4 & 0.0 & -8.1 & -86.8 & -38.6 \\
\hline 5 & 100.0 & 100.0 & 9.1 & 68.9 & 17.3 & 61.3 & 0.0 & -6.9 & -86.8 & -38.7 \\
\hline 6 & 100.0 & 100.0 & 9.0 & 65.1 & 17.0 & 55.9 & 0.0 & -9.3 & -86.9 & -44.1 \\
\hline 7 & 100.0 & 100.0 & 8.8 & 62.7 & 16.8 & 56.5 & 0.0 & -9.7 & -86.9 & -43.5 \\
\hline 8 & 100.0 & 100.0 & 8.7 & 60.6 & 16.6 & 55.8 & 0.0 & -6.8 & -87.0 & -44.2 \\
\hline 9 & 100.0 & 100.0 & 8.6 & 58.8 & 16.4 & 57.7 & 0.0 & -5.7 & -87.0 & -42.3 \\
\hline 10 & 100.0 & 100.0 & 8.6 & 57.2 & 16.2 & 55.4 & 0.0 & -5.5 & -87.0 & -44.6 \\
\hline
\end{tabular}
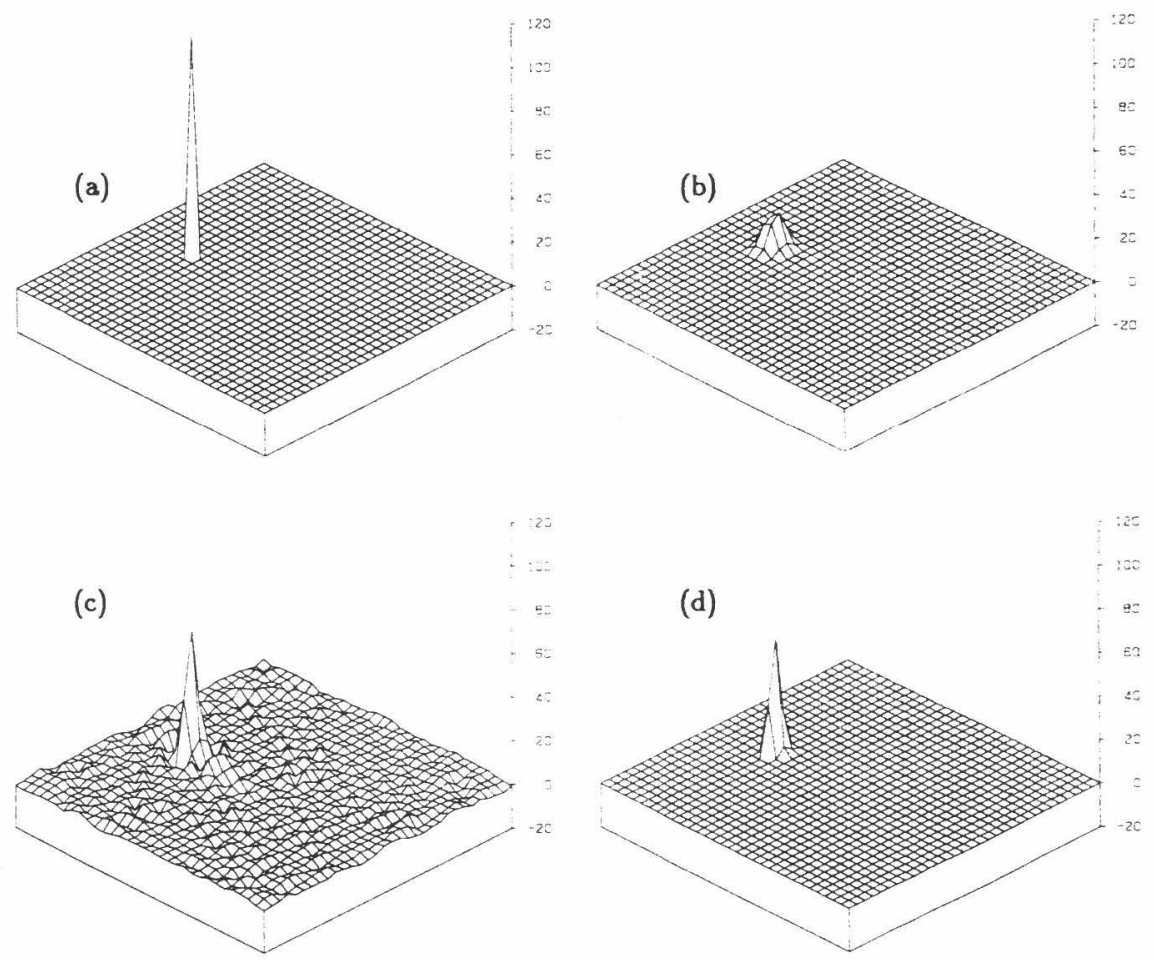

Figure 5. "Delta" function after ten rotations:
(a) Analytical solution.
(b) PDPS method.
(c) PS method.
(d) FPS method. 
Parameters of the numerical solutions after each rotation are presented in Table 3. In the PDPS solution the square of mass is significantly reduced after first rotation $(10.4 \%)$ and then decreases slowly to $8.6 \%$ after 10 rotations. In the PS solution the square of mass is conserved better - $89.1 \%$ after first rotation and $57.2 \%$ after 10 rotations. In case of the "delta" initial condition, the square of mass conservation is closely related to the maximum reduction. In the PDPS solution, the maximum after first rotation is equal to $19.5 \%$ and its location is shifted one grid size from the analytical position: $(8,16)$ - analytical solution, $(9,16)$ - PDPS solution. The same shifting may be observed after the second and next rotations. It does not occur however, in the PS solution. Also, the maximum is significantly higher: 70.2 after the first rotation and 55.4 after 10 rotations.

In the PS solution, the shifting of the maximum is visible in the value of maximum error: -88.9 after first rotation and -87.0 after 10 rotations. In the PS solution, maximum error is lower (in absolute value): $\mathbf{- 2 9 . 7}$ after the first rotation and $-\mathbf{4 4 . 6}$ after 10 rotations.

\section{CONCLUSIONS}

The multi-dimensional filtering procedure presented in this paper is simple and comprehensive both in mathematical formulation and in practical application. It eliminates all negative values from the numerical solution of the advection equation and conserves initial mass by $100 \% \pm 0.0001 \%$. In case of the PDPS solution, additional computer-time needed for filtering does not exceed $4 \%$. The filtering procedure is general enough to be combined with methods other than PS. It can be easily combined with the methods which use explicit time integration algorithms. The filter can also be applied to the numerical solution of the advection-diffusion equation.

Numerical advective tests performed with the PDPS, PS and FPS methods indicate that the results strongly depend on the initial condition.
For both methods, the most commonly used cone-shape initial condition gives very good results with regard to accuracy and short wave noise. In case of rectangular block initial condition, accuracy of both methods is similar, but the PDPS solution does not contain negative concentrations and short wave noise. The "delta" initial condition is difficult for both methods. However, again the short wave noise generated by the PS algorithm is eliminated by PDPS method.

For all tested initial conditions, the filter was also applied directly to the final PS solutions. The results were similar to the PDPS solutions with slightly lower accuracy. This FPS solution can also be recommended for the problems in which only the final concentration field must be free of negative values.

To summarize, the filtering procedure presented in this paper should be a useful tool in the numerical solutions of the advection and advection-diffusion equation. In particular, it can be easily applied to the transport equations in LRTAP models.

\section{ACKNOWLEDGEMENTS}

I would like to thank my colleagues K. Olendrzynski from the Institute of Meteorology and Water Management in Warsaw, R. Shaw and J. Alcamo from the International Institute for Applied Systems Analysis in Laxenburg for valuable comments and discussions. I am also indebted to M. Posch from IIASA who developed the graphical software used in this paper and reviewed the manuscript.

\section{REFERENCES}

[1] Eliassen, A., and Saltbones, J. (1983) Modeling of long range transport of sulfur over Europe: a two-year model run and some model experiments. Atmos. Environ., 17, 1457-1473.

[2] Bottenheim, J.W., Brice, K.A. and Anlauf, K.G. (1984). Discussion of a 
Lagrangian trajectory model describing long-range transport of oxides of nitrogen, the incorporation of PAN in the chemical mechanism, and supporting measurements of PAN and nitrate species at rural sites in Ontario, Canada. Atmos. Environ., 18, 2609-2619.

[3] Zlatev, Z., Berkowicz, R. and Prahm, L.P. (1985), Studying the nitrogen pollution over Europe: I. Description of the model and some simple tests. Air Pollution Laboratory, Danish National Agency of Environmental Protection. Report MST LUFT-A-99.

[4] Carmichael, G.R., Peters, L.K. and Kitada, T. (1986), A second generation model for regional-scale transport (chemistry) - deposition. Atmos. Environ., 20, 173-188.

5] Zlatev, Z. (1988), Treatment of some mathematical models describing longrange transport of air pollutants on vector processors. Parallel Computing., 6, 87-98.

[6] Soong, S.T. and Ogura, Y. (1973), A comparison between axisymmetric and slab symmetric cumulus models. J. Atmos. Sci., s0, 879-893.

[7] Long, P.E. and Pepper, D.W. (1981), An examination of simple numerical schemes for calculating scalar advection. J. Appl. Meteor., 20, 146-156.

[8] Forester K.C. (1977), Higher order monotonic convective difference schemes. $J$. Comput. Phys., 2s, 1-22.

[9] Adam, Y. (1985), Nonlinear instability in advection-diffusion numerical models. Appl. Math. Modeling, 9, 434-440.

[10] Chapman, M. (1981), FRAM - Nonlinear damping algorithms for the continuity equation. J. Comput. Phys., 44, 84-103

[11] Bartnicki, J. (1986), An efficient positive definite method for the numerical solution of the advection equation. IIASA Working Paper WP-86-35. International Institute for Applied Systems Analysis, Laxenburg, Austria.

[12] Pepper D.W., Kern, C.D. and Long, P.E. (1979), Modeling the dispersion of atmospheric pollution using cubic splines and Chapeau functions. Atmos. Environ., 13, 223-237

[13] Raymond W.H. and Garder, A. (1976),
Selective damping in a Galerkin method for solving wave problems and variable grids. Mon. Weath. Rev., 104, 1583-1590.

[14] Gresho P.M. and Lee, R.L. (1981), Don't suppress the wiggles - they're telling you something. Mon. Weath. Rev., 104, 1583-1590.

[15] Orszag, S.A. (1971), Numerical simulation of incompressible flows within simple boundaries. I. Galerkin (Spectral) Representations. Study in Appl. Math., 50, 293-327.

[16] Gottlieb, D. and Orszag, S.A. (1977), Numerical analysis of spectral methods: Theory and Application, Society for Industrial and Applied Mathematics. Philadelphia, Pennsylvania 19103.

[17] Orszag, S.A. (1971), Numerical simulation of incompressible flows within simple boundaries: accuracy. J. Flusid. Mech., 1T, 75-112.

[18] Chock, P.P. (1985), A Comparison of numerical methods for solving the advection equation II. Atmos. Environ., 19, 571-586.

[19] Cooley, J.W. and Tukey, J.W. (1965), An algorithm for the machine calculation of complex Fourier series. Math. Comp., 19, 297-301.

[20] Christensen, O. and Prahm, L.P. (1976), A psendospectral model for dispersion of atmospheric pollutants. J. Appl. Meteor., 15, 1284-1294.

[21] Wengle, H., van den Bosk, B. and Seinfeld, J.H. (1978), Solution of atmospheric diffusion problems by pseudospectral and orthogonal collocation methods. Atmos. Environ., 12, 1201-1032.

[22] Zlatev, Z., Berkowicz, R. and Prahm, L.P. (1983), Three-dimensional advection-diffusion modeling for regional scale. Atmos. Environ., 17, 491-499.

[23] Gazdag, J. (1973), Numerical convective schemes based on accurate computation of space derivatives. J. Comput. Phys., 13, $100-113$.

[24] Dovland, H. and Saltbones, J. (1979), Emissions of sulfur dioxide in Europe in 1978. Norwegian Institute for Air Research Report EMEP/CCC 2/79. 


\section{APPENDIX}

In this Appendix we present the FORTRAN code of the filtering procedure described in this paper.

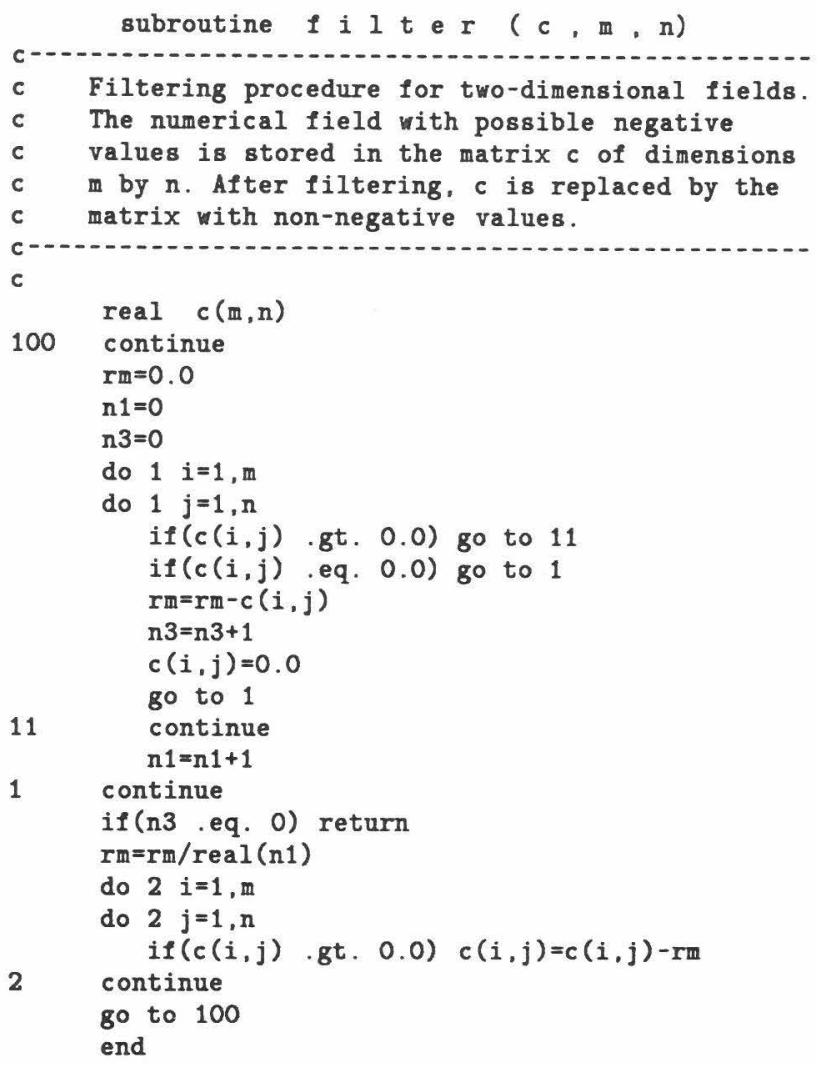


